\title{
ANTICANCER DRUGS
}

\section{Prioritizing targets}

A current priority in cancer research is to identify genetic alterations that are directly involved in tumorigenesis and develop therapies to target them. Arul Chinnaiyan and colleagues have used a prioritization strategy to show that angiotensin II receptor type I (AGTR1) is overexpressed in a subset of breast cancers and that these are sensitive to an AGTR antagonist.

Chinnaiyan and colleagues reasoned that genes which show the largest changes in expression in cancer (ranging from a 10-fold to over a 100-fold increase relative to baseline expression) might have a direct role in cancer progression. They combined two approaches that have previously been separately applied to identify cancer genes

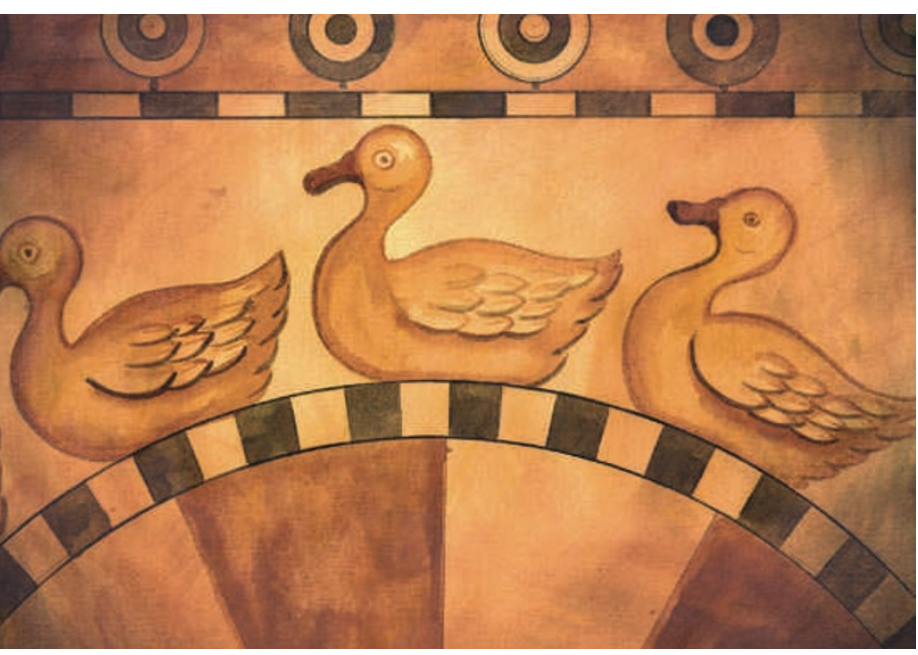

- cancer outlier profile analysis and meta-analysis. Using this new method to analyse 31 breast cancer profiling data sets, which comprised $\sim 3,200$ microarray experiments, they identified 159 genes that were overexpressed in a significant fraction of the data sets (meta-outliers).

The authors found that ERBB2 was the most significant meta-outlier, consistent with the fact that this gene is overexpressed in 25-30\% of breast tumours. The next most significant meta-outlier was AGTR1, which has previously been linked to cancer. AGTR1 overexpression was restricted to a subset of oestrogen receptorpositive tumours and was mutually exclusive of $E R B B 2$ overexpression.

What is the function of AGTR1 overexpression in breast cancer? Rhodes et al. used an adenovirus construct to overexpress AGTR1 in H16N2 and HME human mammary epithelial cell lines and assayed the cells for proliferation and invasion in serum-free media or on stimulation with angiotensin II, the ligand of AGTR1. They found that overexpression of AGTR1 alone or on stimulation had no effect on proliferation, but overexpression of AGTR1 combined with angiotensin II stimulation significantly promoted cell invasion in both cell lines. When the authors treated the cells with losartan, an AGTR1 blocker that is approved for treating hypertension, they found that the AGTR1-mediated invasion phenotype was attenuated in a dosedependent manner. Moreover, when they stimulated breast cancer cell lines that overexpressed AGTR1 with angiotensin II, they found an increase in invasion, which was also reversible by addition of losartan.

The authors then tested the effects of overexpressing AGTR1 in MCF7 breast cancer xenografts in mice. They found that MCF7-AGTR1 tumours were of a similar size as control MCF7 tumours at 2 weeks or 8 weeks after the xenografts were established. However, administration of losartan specifically reduced the growth of MCF7-AGTR1 tumours at both of these time points relative to the control xenografts. These results suggest that AGTR1 does not synergize with the activating PI3K mutation present in MCF7 cells to provide an additive growth signal, but it does sensitize tumours to growth inhibition on treatment with losartan. Therefore, this study suggests that targeted therapy with losartan may be beneficial in patients with breast cancers that are positive for AGTR1.

Meera Swami, Nature Reviews Cancer

ORIGINAL RESEARCH PAPER Rhodes, D. R. et al. AGTR1 overexpression defines a subset of breast cancer and confers sensitivity to losartan, an AGTR1 antagonist. Proc. Natl Acad. Sci. USA 1 Jun 2009 (doi: 10.1073/pnas.0900351106) 